\title{
Application of PIP data in health economic models for market access
}

\author{
Nadine van Dongen' \\ Mark JC Nuijten ${ }^{2}$ \\ 'Van Dongen Research Ltd, \\ London, UK; ${ }^{2}$ Ars Accessus Medica, \\ Amsterdam, The Netherlands
}

Correspondence: Nadine van Dongen Van Dongen Research Ltd, London, UK Tel +44754522 3534

Email nadine.vandongen@vandresearch.com
This article was published in the following Dove Press journal:

Patient Intelligence

23 November 2010

Number of times this article has been viewed

\begin{abstract}
Cost-effectiveness data from a state of the art health economic analysis should permit reliable, reproducible, and verifiable insights into the effectiveness of a drug and the possible savings that might be achieved relative to other drugs and/or treatments. The data for a model may come from a variety of sources and are subject to varying degrees of uncertainty. The reliability of the estimates depends on the choice of the data sources. Data sources for the variables being used in a model may be clinical trials, databases, medical records, and Delphi panels. A limitation of these data sources is that they often lack the input from the patient's perspective. Patient Intelligence applications can provide data to be used in health economic models for any given situation regarding treatment of persons suffering from a disorder, disease, or complaint. The objective of this paper to explore the opportunity of integrating patient data generated by Patient Intelligence applications as an alternative data source for a Delphi panel and databases in health economic models.
\end{abstract}

Keywords: effectiveness, Patient Intelligence, data source, Delphi panel

\section{Introduction}

Reimbursement of new pharmaceuticals up until now has been based merely on registration data (efficacy, safety, and quality parameters). However, escalating costs have become a major concern for health care decision-makers, prompting the implementation of new cost containment measures, leading to additional data requirements for new pharmaceuticals, which especially relate to the use of innovative medication in real daily practice. ${ }^{1}$ The most important new data requirements are effectiveness, cost-effectiveness, and budgetary impact. Effectiveness offers a picture of the actual value of an innovation in daily practice. ${ }^{2}$ Looking at efficacy, the effect is examined under ideal conditions in a homogeneous group of patients, and usually with the assistance of intermediate (surrogate) endpoints. Effectiveness data, on the other hand, offer a clearer picture of the actual value because the effect is examined under more realistic conditions using a more heterogeneous group of patients.

Cost-effectiveness data from a state of the art health economic analysis should permit reliable, reproducible, and verifiable insights into the effectiveness of a drug and the possible savings that might be achieved relative to other drugs and/or treatments. Because economic evaluation will play a fundamental role in health care decision-making, it is vital that the methods used in such evaluations are carefully scrutinized and refined. If economic analysis is to play an important and useful role in the allocation of scarce health care resources, then such analyses must be performed properly and with care. ${ }^{1}$ 
In most clinical trials, economic data are not collected alongside the study. Even when they are, the data may need to be projected to populations, time periods, or settings that were not observed in the clinical study. In these cases, decision-analytic models may provide some of the missing information. The model resulting from the decision analysis must correspond, as far as possible, to the real-life situation of the disease and should reflect actual treatment patterns, with input values (probabilities and items of health care utilization) deviating as little as possible from population values. ${ }^{3}$ The data for a model may come from a variety of sources and are subject to varying degrees of uncertainty. The reliability of the estimates depends on the choice of the data sources (selection criteria, external validity) and data processing (calculation of mean or median, Mantel-Haenszel method, actuarial method). Hence, there may be a potential bias, both in the choice of the data sources and the methods of data processing. Data sources for the variables being used in a model may be clinical trials, literature (eg, meta-analysis), databases, medical records, Delphi panels, patient panels, and/or official tariff lists for health care use. ${ }^{4}$ A limitation of these data sources is that they lack the input from the patients' experience and perspective and often suffer from limited external validity. Especially with regards to integrating resource utilization and patient outcomes, like health-related quality of life, the patient's voice is required because patients themselves are the key determinants in the clinical and economic outcomes of treatment in daily life and, as a consequence, patients can provide data with high external validity, fulfilling one of the most important requirements for health economic studies.

Patient Intelligence refers to skills, technologies, applications, and practices used to help an organization acquire a better understanding of its position in the health care context. Patient Intelligence may also refer to the information collected by patients on resource utilization and quality of life. Patient Intelligence applications provide historic and current views of any given present situation regarding behavior and treatment of persons suffering from a disorder, disease, or complaint. Patient Intelligence is often aimed at the support of better decision-making in the health care environment. Thus, a Patient Intelligence system can be called a decision support system.

The objective of this paper was to explore the opportunity of integrating Patient Intelligence applications, like Patient Intelligence Panel (PIP) data, as an alternative data source for a Delphi panel and databases in health economic studies.

\section{Current data sources Delphi panel}

The use of expert opinion is appropriate in situations in which there is little or no published material in a particular area, or in which the results of a thorough literature review or meta-analysis are considered unreliable, ${ }^{5}$ conflicting, ${ }^{6}$ or insufficient to cover the requirements of a study. Delphi panels operate in stages or rounds, in an effort to obtain a convergence of expert opinion in a particular area. The main areas of weakness are as follows. A problem related to the iterative process in the Delphi method is the potential for experts to drop out following the first round. This behavior may lead to a response bias if the attrition rate is substantial. In pharmacoeconomic studies reviewed, there are no explicit criteria for the selection of experts for participation in the studies. ${ }^{7}$ The use of a "physician-expert panel" to estimate resource use, while common, carries the risk that respondents may give inaccurate estimates or specify the resources required for ideal care, rather than that provided in practice. Physicians may adjust estimations based on other estimations, because they do not want to be outliers. In addition, physicians may overestimate variables related to the success of their treatment (for example, response rates, mortality, complications, adverse events), and also underestimate variables related to health care use, in order to be more efficient. The most important weakness of the Delphi panel is that the data are not real, but only estimates.

\section{Static databases}

The two different databases that can be distinguished are claims databases and clinical outcomes databases. In the case of claims databases, the objective is to collect, for administrative purposes, all data on health care resources used, whereas in clinical databases, the objective is to measure clinical outcomes for medical or scientific purposes. In both cases the databases can be considered to be static because the information in the databases are accumulated facts on demographics, treatments, and endpoints, which may not correspond with the specific data requirements of a health economic model. A database may not be a suitable source for guiding decisions in health care because so much of the data it contains is not scientifically valid. Although databases may contain a lot of detailed information on both clinical and economic outcomes, the format of this information has to fit the structure of the health economic model. However, the majority of the existing databases have not been developed for economic evaluations. ${ }^{4}$ 
The patients in databases are usually labeled according to existing medical classification systems, such as the International Classification of Diseases, Ninth Revision, Clinical Modification (ICD-9-CM) or diagnosis-related groups for inpatient care. Because of financial incentives, patients may be labeled with a different ICD-9 code or a more severe diagnosisrelated group. Another problem associated with diagnosis is the lack of consensus on defining criteria for a pathology, and the overlap of symptoms. For example, in psychiatric disorders, patients with depression and those with generalized anxiety disorder frequently have overlapping characteristics, which makes it difficult for a physician to distinguish clinically among these disorders. Hence, a subpopulation of patients with ICD-9-IM code for depression may include patients with generalized anxiety disorder because of inaccurate diagnosis.

The population in a claims database may not be representative of the overall population and may contain a biased sample of patients. For example, in the US, Medicare databases will only consist of the elderly, while Medicaid databases will only contain patients of low sociodemographic status. ${ }^{8}$

Clinical databases contain mainly clinical outcomes, but usually no, or incomplete, information on health care use. The external validity may be limited because of bias resulting from the site(s) of data collection, the specialty of the physicians, and the primary objective of the database implementation, which may vary between a prospective clinical study and evaluation of a health care program. Prospective studies have the same limitations as clinical trials, while for a health care program, the limited choice of therapy will reduce the external validity. ${ }^{8}$ The clinical outcomes should have enough external validity in order to be used as an effectiveness measure or to be extrapolated to effectiveness measures of direct relevant outcomes (quality of life and quality-adjusted survival [Q-TWIST], quality-adjusted life years, or quality of life) for pharmacoeconomic studies are usually not included.

\section{Patient Intelligence Panel}

The PIP is an online patient panel that gives researchers access to patients worldwide. An online panel database (Internet access panel) is a group of prescreened respondents who have expressed a willingness to participate in surveys. ${ }^{9}$ Respondents become "panelists" by completing a profiling questionnaire. The data collected in the profiling includes demographics and health status characteristics. The panel can range in size from 100 to 100,000 or more people. By having access via the Internet to thousands of people around the world who are willing to participate in research on health care and specific indications, all questions can be asked and a wide range of direct patient data can be obtained. PIP data can be collected specifically on economic (resource use) as well as clinical, patient-reported outcomes, and quality of life outcomes to be used as input for a health economic model and therefore perfectly fulfilling the requirement of input data, with high external validity for a health economic model.

As mentioned before, online panel research makes it possible to gather data for hundreds of patients within seven days. Previous PIP research implemented in 2009 included 300 patients diagnosed with depression and taking different antidepressants. They were asked to quantify the severity of side effects encountered when taking antidepressants, and they were asked to identify each side effect occurring, and then rate this side effect according to severity on a scale of 1 to 10 (10 being most severe, ${ }^{10}$ see Figure 1). By quantifying a patient's perception of such treatment, data with a high

\section{PIP output: Severity of side effects}

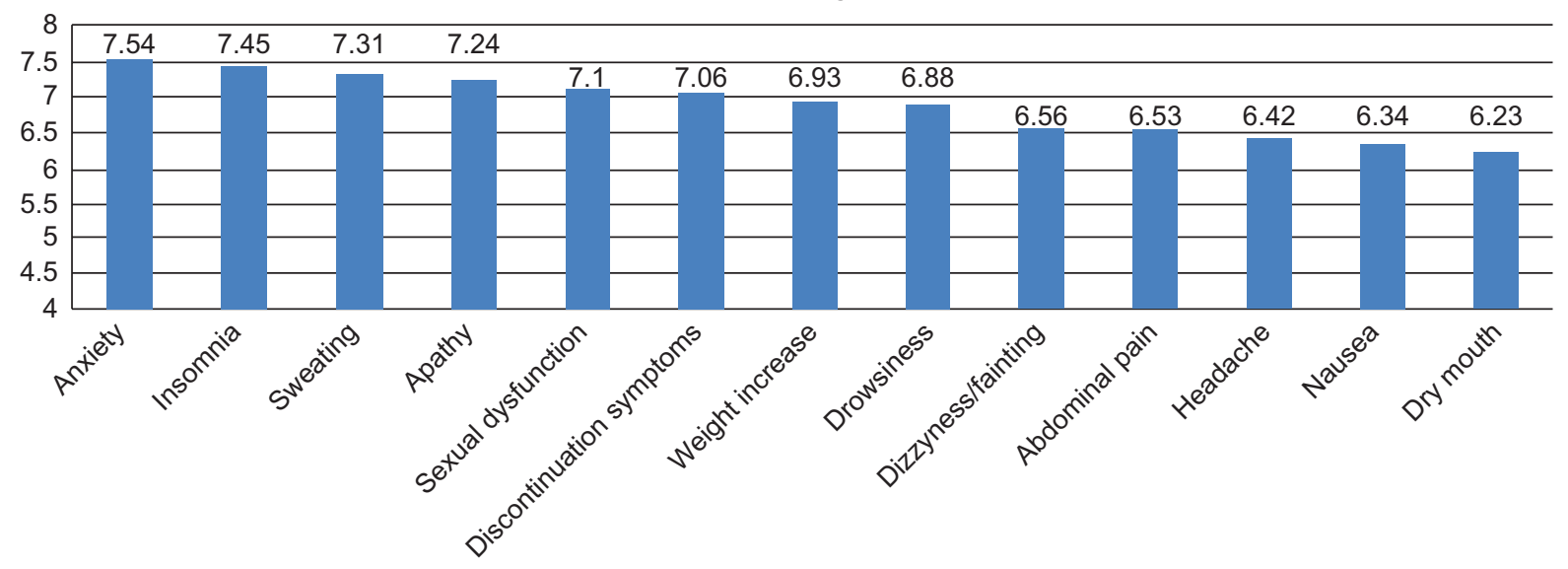

Figure I Patient perceptions in treatment of depression. Abbreviation: Patient Intelligence Panel. 
external validity representing daily practice can be collected and integrated as a patient outcome in a health economic model.

A PIP study can be designed to measure the impact of a particular disease or condition on clinical and patient-specific outcomes, and to document the outcomes associated with different treatments or settings of care in a quantitative matter. Patients can be followed prospectively, and data are collected on disease severity and clinical outcomes as reported by clinicians, as well as resource use, functional status, and quality of life (patient outcomes) as reported by the patient. PIP data reflect the current treatment patterns without influencing the treatments or interventions, and consequently the PIP study is fully naturalistic without any intervention with real practice (eg, no randomization) and has a high external validity. The PIP data can yield real-life data for the comparator in the health economic model. The PIP may also yield more statistically solid safety data in real life with a high external validity because of the large sample size of the PIP. The large sample size of the PIP may also allow the identification of any type of covariance, which could not be proven in a clinical trial because of lack of power. As a consequence, a PIP study has the power for the development of statistically solid multiple regression equations with high external validity, which can be incorporated in a health economic model. A KOL (Key Opinion Leaders) validation process can be integrated after statistically relevant outcomes of the PIP patient's feedback. Besides that, it is prudent to include KOLs in the survey conception, this allows online surveys to be validated from a health care professional point of view, and therefore adding another dimension to the outcomes.

Summarizing, the cost-effectiveness of a new product can be modeled by combining data on the probabilities derived from the literature and clinical trials with information on costs and utilities from a PIP, which is primarily developed for the collection of health economic data in daily practice. Thus, a PIP data set yields high external validity and its large sample size also reduces the statistical constraints of smaller study designs, like a cross-sectional study.

\section{Discussion}

A comparison of the PIP data set versus the Delphi panel is in favor of the PIP data set, because the Delphi panel is based on estimates only and the PIP database comes from real data. In addition, the Delphi panel suffers from methodologic limitations, as described earlier, which may lead to bias in the estimates.
A comparison of the PIP data set versus static databases is more comprehensive. At first glance, the most important similarity is that both data sets are based on actual data, and thereby overcome the limitations of the Delphi panel, but a lot of differences between the PIP data set and database can be noticed.

The PIP data set is not limited by power constraints as are static databases, and especially clinical databases, which usually have a limited number of patients. Claims databases usually have a large number of "patients", but the absolute number is fixed. The sample size of the PIP data set can be adjusted based on a priori sample size calculations in order to show statistical significant results. The opportunity to adjust the sample size also allows the development of regression equations and the application of other advanced statistical methods for handling bias, confounding variables, and covariates using PIP.

The prospective design of the PIP data set allows the a priori definition of all the economic and clinical variables required for the health economic model, and which will fulfill the technical requirements of the model. In contrast, database studies are usually retrospective, which means that not all data may be collected or data may not fully correspond with the requirements of the model. The prospective use of a database allows more opportunities for collecting the appropriate data in the right format, but a database is developed for another purpose and therefore the use of database data can be questioned from a strictly scientific point of view. On the other hand, a PIP data set can be primarily developed for a cost-effectiveness study, which is in line with state of the art research, where outcomes and data collection should be primarily driven by the research question. Finally, most databases, especially claim databases, do not contain clinical outcomes (quality of life and quality-adjusted survival [Q-TWIST], quality-adjusted life years, or quality of life, patient satisfaction, patient-reported outcomes, or noncompliance), which are essential for a cost-effectiveness study. The PIP data design allows the collection of all relevant clinical outcomes, including outcomes from a patient perception, which cannot be derived from a database, whereas these outcomes are the ultimate input data for a cost-effectiveness model because of its high representativeness and external validity.

A problem associated with databases can be the lack of consensus on defining criteria for a pathology, and the overlap of symptoms, as was mentioned earlier. The PIP data set can be used to define exactly the number of 
patients who need to be included (or excluded) from the health economic model, including criteria for comorbidity or risk factors. The databases usually contain limited sociodemographic data, but the PIP data set can collect all relevant sociodemographic information necessary for a cost-effectiveness model.

When data are missing in a database, when queries are raised, or when additional information is needed, these issues are unable to be resolved in a database study. It is not easy to go back directly to individual patients, because of both database structure and legal issues related to privacy of the patient. The PIP data set allows a direct link to individual patients for collecting additional information without any constraints. Patients in a PIP can be identified and are traceable. This is not only important for filling the actual costeffectiveness model with data, but also for a validation of the cost-effectiveness model after three years. For example, some countries require prospective follow-up after submission of the model in order to update the model later with real-life data after launch of a new medication. This means that the PIP data set can be used for long-term follow-up. Important measures in this validation phase are quality of life and patient satisfaction, which are typical outcomes of the PIP data set.

The PIP is based on the infrastructure of the Internet and information technology so can work quicker than a database, where there are limitations of access, privacy data, and underlying database data abstraction, which require additional software and programming.
The PIP database is funding patient's organizations and therefore patients do not have a financial incentive, which increases its scientific credibility. However, database studies often require substantial funding by the database owner, which may lead to a conflict of interest. In addition, labeling of patients can be biased by financing incentives for the physicians, like overgrading of diagnosis-related groups leading to a higher fee. The Table 1 summarizes the main differences between the data sources, as described.

\section{Conclusion}

With the growing importance of modeling studies for economic evaluations, a new area for research has been created. In order to obtain objective and reproducible results from such studies, it is important to have standardized methods of evaluation contained in accepted guidelines on methodology. This paper showed that when integrating patient data in models, a more holistic outcome will be the result, corresponding with the concept of costeffectiveness requiring high external validity and outcomes representing real life. Patient data can be considered the optimal data source for a health economic model because it has the highest representativeness of the effectiveness of a treatment in real life. Especially for perception of sensitive factors in health economic models, like quality of life, side effect severity, and rationale for discontinuation, the patients' perception should be integrated because the patient is the sole source for outcomes related to the patient's experience with pharmaceutical therapy. Finally, the use of Patient

Table I Comparison data sources for a cost-effectiveness model: quality of data

\begin{tabular}{|c|c|c|c|c|}
\hline & & Delphi panel & Static database & Patient panel \\
\hline Up till now: & Efficacy & low & low & medium \\
\hline \multirow[t]{2}{*}{ registration data } & Safety & low & medium & high \\
\hline & Quality parameters & low & medium & medium \\
\hline \multirow[t]{15}{*}{ Today } & Effectiveness (innovation value) & low & medium & high \\
\hline & Cost effectiveness & low & low & high \\
\hline & PRO (patient reported outcome) & low & low & high \\
\hline & eg, satisfaction & & & \\
\hline & Quality of Life & low & low & high \\
\hline & Compliance & low & medium & high \\
\hline & Cost effectiveness & low & medium & high \\
\hline & Budgetary impact & medium & high & high \\
\hline & Real life situations & medium & medium & high \\
\hline & Reliability & low & medium & high \\
\hline & Reproducibility & low & medium & high \\
\hline & Variable insights & medium & high & high \\
\hline & Compliance/adherence & medium & medium & high \\
\hline & Actual treatment patterns & medium & high & high \\
\hline & Speed of data collection & high & low & high \\
\hline
\end{tabular}


Intelligence research suits perfectly the concept of evidencebased medicine, which means that clinical encounters should be supported by scientific conclusions based on real data as far as possible.

\section{Disclosure}

The authors report no conflicts of interest in this work.

\section{References}

1. Nuijten MJ, Mittendorf T, Persson U. Practical issues in handling data input and uncertainty in a budget impact analysis. Eur J Health Econ. 2010 Apr 3. [Epub ahead of print].

2. Drummond MF, Schwartz JS, Jönsson B, et al. Key principles for the improved conduct of health technology assessments for resource allocation decisions. Int J Technol Ass in Health Care. 2008;24(3):244-258.
3. Weinstein MC, Fineberg HV. Clinical Decision Analysis. Philadelphia, PA: WB Saunders Co; 1980.

4. Nuijten MJ. Bridging decision analytic modelling with a cross-sectional study. Application to Parkinson's disease. Pharmacoeconomics. 2000;17(3):227-236.

5. Jariath N, Weinstein J. The Delphi methodology (Part two): A useful administrative approach. Can J Nurs Adm. 1994;7(4):7-20.

6. Jones J, Hunter D. Consensus methods for medical and health services research. BMJ. 1995;311(7001):376-380.

7. Evans C. The use of consensus methods and expert panels in pharmacoeconomic studies: Practical applications and methodological shortcomings. Pharmacoeconomics. 1997;12 (2 Pt 1):121-129.

8. Nuijten MJC. In search for more confidence in health economic modelling. Thesis. 2003. Erasmus University Rotterdam.

9. van Dongen N. Let's be effective, let the patients talk! Does 'patient intelligence' have an effect on improvements in quality within the healthcare environment? Patient Intelligence. 2009;1:1-5.

10. van Dongen Research. Data on file. 2009.
Patient Intelligence

\section{Publish your work in this journal}

Patient Intelligence is an international, peer-reviewed, open access journal that characterizes and measures the central role of patient behavior and intention in optimizing healthcare management in all areas of disease and complaint types. An improved understanding of patient intelligence coupled with predictive analysis helps an organization contribute more effectively to achieving better outcomes.
Dovepress

The journal is characterized by the rapid reporting of reviews, original research, methodologies, analytics, modeling, clinical studies and patient surveys across all disease areas. The manuscript management system is completely online and includes a very quick and fair peer-review system. Visit http://www.dovepress.com/ testimonials.php to read real quotes from published authors. 